\title{
A Galileo IOV assessment: measurement and position domain
}

\author{
Ciro Gioia • Daniele Borio • Antonio Angrisano • \\ Salvatore Gaglione $\cdot$ Joaquim Fortuny-Guasch
}

Received: 4 September 2013/Accepted: 16 April 2014/Published online: 24 June 2014

(C) The Author(s) 2014. This article is published with open access at Springerlink.com

\begin{abstract}
The European GNSS, Galileo, is currently in its in-orbit validation (IOV) phase where four satellites are finally available for computing the user position. In this phase, the analysis of the measurements and position velocity and time (PVT) obtained from the IOV satellites can provide insight into the potentialities of the Galileo system. A methodology is suggested for the analysis of the Galileo IOV pseudorange and pseudorange rates collected from the E1 and E5 frequencies. Several days of data were collected and processed to determine figures of merit such as root mean square and maximum errors of the Galileo observables. From the analysis, it emerges that Galileo is able to achieve better accuracy than GPS. A thorough analysis of the PVT performance is also carried out using broadcast ephemerides. Galileo and GPS PVTs are compared under similar geometry conditions showing the potential of the Galileo system.
\end{abstract}

Keywords Accuracy - Galileo · In-orbit validation . IOV · Multi-frequency · Position velocity and time - PVT . Precise orbits - Pseudorange Pseudorange rate

\section{Introduction}

Galileo, the European Global Navigation Satellite System (GNSS), is currently in its in-orbit validation (IOV) phase,

C. Gioia $(\bowtie) \cdot$ A. Angrisano $\cdot$ S. Gaglione

Department of Science and Technology,

Parthenope University of Naples, Naples, Italy

e-mail: ciro.gioia@jrc.ec.europa.eu

C. Gioia - D. Borio · J. Fortuny-Guasch

Institute for the Protection of the Citizen (IPSC),

Joint Research Centre (JRC) of the EC, Ispra, VA, Italy and four satellites are finally available for computing the user position. The availability of the Galileo quartet allows researchers to investigate the potentiality of the Galileo system and extend previous results based on the signals broadcast by the Galileo in-orbit validation element (GIOVE) experimental satellites. More specifically, although the two GIOVE satellites did not allow the computation of the user position, it was possible to test the performance of new acquisition and tracking algorithms designed to fully exploit the benefits of the new Galileo signals. GIOVE satellites also allowed researchers to assess the ranging capabilities of future Galileo signals (Angrisano et al. 2012) and provided a significant experience for the design of next-generation Galileo satellites.

The first two IOV satellites were launched in October 2011, whereas the satellite quartet required for positioning was completed 1 year after, in October 2012. In the same year, GIOVE-A and GIOVE-B were decommissioned. The four IOV satellites transmit signals on E1, E5 and E6 bands, and Galileo-only positioning has been possible since March 2013, when the European Space Agency (ESA) started disseminating valid Galileo ephemerides. Although Galileo ephemerides are still broadcast in a discontinuous way and without warranty, they allow position computation using the sole Galileo signal-in-space (SIS). Since March 2013, several research groups reported successful Galileoonly positioning including the authors (Angrisano et al. 2013).

Despite the race to demonstrate Galileo-only positioning, limited analysis has been performed to evaluate the accuracy of the measurements broadcast from Galileo satellites. In particular, ranging capabilities of the IOV satellites can be assessed employing the precise orbits determined using the approach described in (Steigenberger et al. 2011) and available from ftp://cddis.gsfc.nasa.gov/ 
pub/gps/products/mgex. These orbits, the availability of ionospheric corrections and a professional receiver placed in a surveyed location can be used to determine figures of merits such as the root mean square (RMS) and the maximum error of the Galileo observables, i.e., pseudoranges (PR) and PR rates (Angrisano et al. 2013).

A methodology is suggested for the analysis of the Galileo IOV PRs and PR rates collected from the E1BC and E5a frequencies. The methodology is an extension of the techniques developed in (Angrisano et al. 2012) and employed to characterize the PRs of GIOVE satellites. With respect to (Angrisano et al. 2012), the following elements of innovation have been introduced: Precise ephemerides are used to obtain a more accurate satellites position, and global ionospheric maps (GIM) are adopted to compute the ionospheric delay. Finally, the analysis is extended to PR rates and to E5a observables.

Several hours of data were collected using a Septentrio PolarRxS receiver and used to characterize the quality of Galileo observables. From the analysis, it emerges that Galileo measurements have accuracies comparable with those of GPS, showing the potential of the European GNSS.

It is noted that, although several groups focused on Galileo-only or combined Galileo-GPS positioning, no result has been published on the accuracy of IOV measurements. These results along with the methodology proposed are the main contributions of this paper. In addition to this, the benefits of combined GPS-Galileo positioning are also highlighted and some considerations on Galileoonly location are provided. More specifically, results obtained using Galileo broadcast ephemerides are also analyzed.

The analysis presented here is an extension of the conference contribution (Angrisano et al. 2013) published by the authors. With respect to the conference paper, a more thorough analysis of the IOV measurements and Galileo position have been performed, including results from the E5a band which was not previously considered. Additional analysis, using single differences (SD), has also been carried out to investigate the contribution of thermal noise. Moreover, the sample results presented were obtained considering a different GPS week from the one provided in (Angrisano et al. 2013). For this reason, the results presented in the following are complementary to those analyzed in (Angrisano et al. 2013).

The navigation states and the methodology developed for the analysis are described in the "Methodology" Section. The experimental setup adopted is detailed in the third section, and experimental results relative to the Galileo observables are described in the fourth section. Galileo PVT performance is discussed in the PVT analysis section, and some conclusions are finally drawn in the last section.

\section{Methodology}

In this section, a detailed description of the methodology proposed is provided. Two different approaches are adopted for the position and the measurement domain analyses. First, the methodology used to evaluate the performance of Galileo single-point positioning is described, and then the multi-constellation approach is illustrated. In the second part of the section, the methodology for evaluating the measurement accuracy is proposed.

\section{Position domain}

GNSS receivers are able to provide three types of measurements: PR, Doppler and carrier phase; only PR and Doppler measurements are considered whose equations are:

$$
\begin{aligned}
& \rho=d+c d t_{u}+\varepsilon_{\rho} \\
& \dot{\rho}=\dot{d}+c \dot{d} t_{u}+\varepsilon_{\dot{\rho}}
\end{aligned}
$$

where $\rho$ is the PR measurement; $d$ is the satellite-receiver distance; $c d t_{u}$ is the receiver clock offset scaled by the speed of light $c$; and $\varepsilon_{\rho}$ contains the residual errors after atmospheric and satellite-related corrections (HoffmannWellenhof et al. 1992, Kaplan and Hegarty 2006, IS-GPS$2002004) \rho$ is the PR rate measurement obtained from Doppler; $\dot{d}$ is the geometric distance rate of change; $c \dot{d} t_{u}$ is the receiver clock drift scaled by the speed of light; and $\varepsilon_{\dot{\rho}}$ accounts for residual errors.

The receiver position, velocity and the clock parameters, computed using trilateration technique starting from PR and $\mathrm{PR}$ rate observations, are computed using the weighted least squares (WLS) considering a weighting matrix related to the satellites elevation (Kaplan and Hegarty 2006). The state vector components are:

$$
\begin{aligned}
& \underline{x}=\left[\begin{array}{ll}
\underline{P} & c d t_{u}
\end{array}\right] \\
& \underline{v}=\left[\begin{array}{ll}
\underline{V} & c \dot{d} t_{u}
\end{array}\right]
\end{aligned}
$$

Equations (1) and (2) are valid when a single GNSS is used, and all the measurements refer to a common time scale. When a GPS and Galileo multi-constellation is considered, additional unknowns have to be included in the state vectors (2), in order to consider the bias and drift between the time scales adopted by the two systems (Fig. 1).

The time difference between GPS and Galileo is broadcast within the Galileo navigation message, and the use of parameters such as the Galileo/GPS time offset (GGTO) (OS-SIS-ICD 2010) will allow the alignment of the GPS and Galileo time scales. Galileo ephemerides are currently transmitted in a discontinuous way, and the GGTO is seldom available. A solution is the inclusion of 


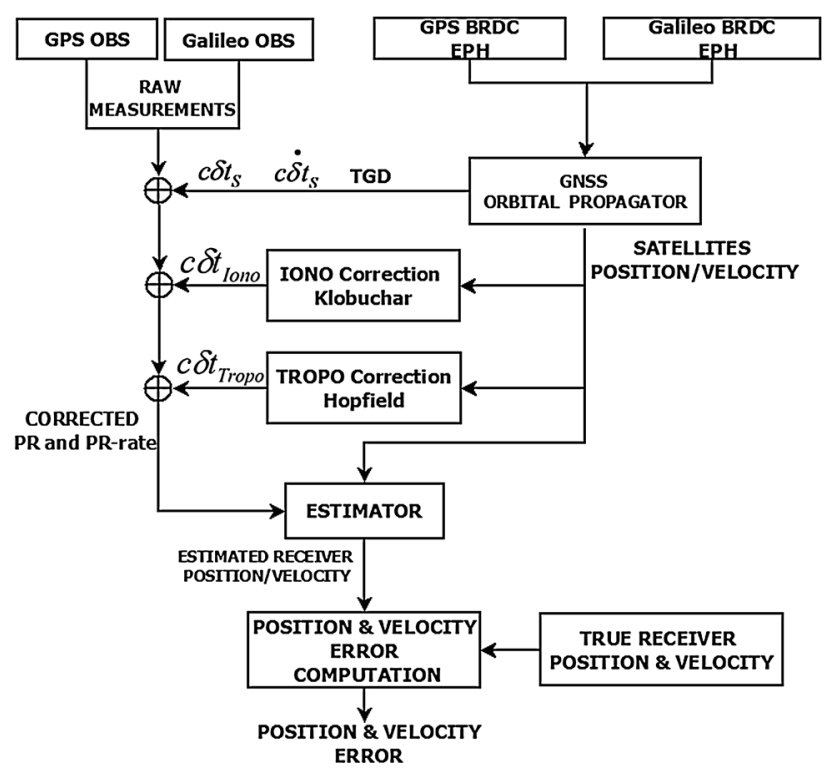

Fig. 1 Schematic representation of the algorithm developed for the determination of position and velocity errors

two additional unknowns representing the bias and drift between GPS time and Galileo system time (GST). The position and velocity state vectors become:

$\underline{x}=\left[\begin{array}{lll}\underline{P} & c d t_{u}^{\mathrm{GPS}} & c d t_{\mathrm{GAL}}^{\mathrm{GPS}}\end{array}\right]$

$\underline{v}=\left[\begin{array}{lll}\underline{V} & c \dot{d} t_{u}^{\mathrm{GPS}} & c \dot{d}_{\mathrm{GAL}}^{\mathrm{GPS}}\end{array}\right]$

where $c d t_{\mathrm{GAL}}^{\mathrm{GPS}}$ and $c \dot{d}_{\mathrm{GAL}}^{\mathrm{GPS}}$ are the bias and drift between GPS time and GST, respectively. Note that also the PR and PR rate models (1) need to be modified in order to account for additional clock terms. In particular, when Galileo measurements are considered, $c d t_{\mathrm{GAL}}^{\mathrm{GPS}}$ and $c \dot{d} t_{\mathrm{GAL}}^{\mathrm{GPS}}$ are added to the PR and PR rate models, respectively. Models (2) and (3) will be used to compute and analyze Galileo-only and combined GPS-Galileo navigation solutions, and the flow chart of the algorithms developed is shown in Fig. 2.

If the user position and velocity are known, the state vector (2) can be reduced only to the clock parameters:

$\underline{x}=\left[\begin{array}{ll}c d t_{u}^{\mathrm{GPS}} & c d t_{\mathrm{GAL}}^{\mathrm{GPS}}\end{array}\right]$

$\underline{v}=\left[\begin{array}{ll}c \dot{d} t_{u}^{\mathrm{GPS}} & c \dot{d} t_{\mathrm{GAL}}^{\mathrm{GPS}}\end{array}\right]$

In this approach, all the measurements are used to estimate the clock unknowns, providing a better estimation of such parameters. The user position and velocity can also be used to determine true geometric distances and distance rates which, in turn, can be adopted to evaluate PR and PR rate errors along with the clock estimates and precise corrections available from ftp://cddis.gsfc.nasa.gov/pub/gps/products/ mgex. A detailed representation of the technique adopted for the evaluation of PR and PR rate errors is depicted in Fig. 2. A more detailed explanation of the methodology adopted can be found in (Angrisano et al. 2013).

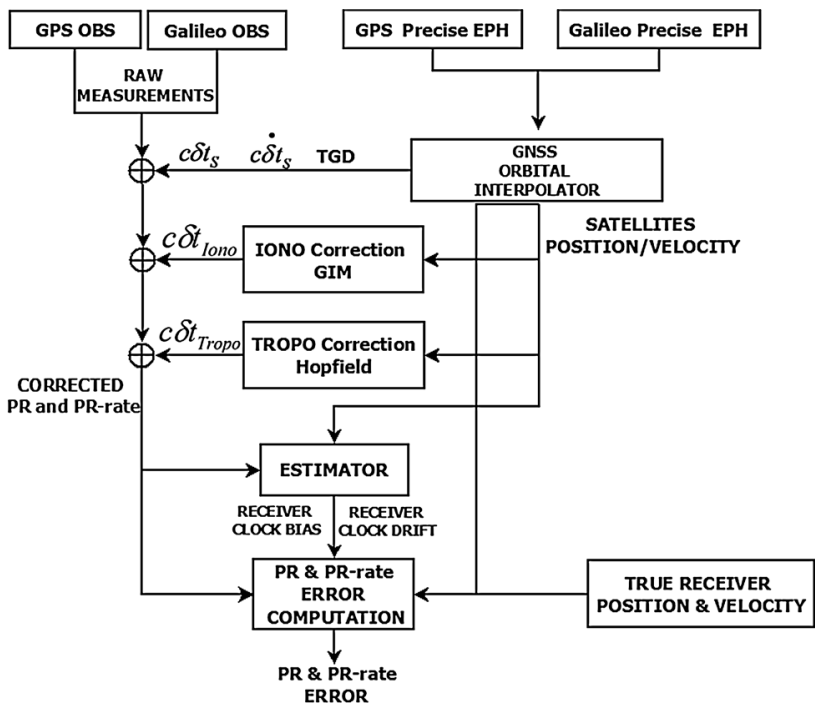

Fig. 2 Schematic representation of the algorithm developed for determining PR and PR rate residual errors

In order to compute Galileo PR and PR rate error for E1 frequency, GPS and Galileo observations are used together; raw $\mathrm{PR}$ and $\mathrm{PR}$ rate measurements are corrected for the satellite clock errors, relativistic effects, the Sagnac effect and atmospheric delays according to:

$$
\begin{aligned}
\rho_{c}= & \rho+c d t_{\mathrm{sv}}-c T_{\mathrm{GD}}-c d t_{u}+c d t_{r}+c d t_{\mathrm{sag}} \\
& -d_{I}-d_{T}-c d t_{\mathrm{GAL}}^{\mathrm{GPS}} \\
\dot{\rho}_{c}= & \dot{\rho}+c \dot{d} t_{\mathrm{sv}}-c \dot{d} t_{u}+c \dot{d} t_{\mathrm{sag}}-c \dot{d} t_{\mathrm{GAL}}^{\mathrm{GPS}}
\end{aligned}
$$

where $c d t_{\mathrm{sv}}$ and $c \dot{d} t_{\mathrm{sv}}$ are the satellite clock bias and drift; $c T_{\mathrm{GD}}$ is the time group delay scaled by the speed of light; $c d t_{r}$ is the relativistic correction; $c d t_{\mathrm{sag}}$ and $c \dot{d} t_{\mathrm{sag}}$ are the Sagnac effect corrections; $d_{I}$ is the ionospheric correction computed using GIM; and $d_{T}$ is the tropospheric correction computed using the Hopfield model; a combined GPS/ Galileo navigation algorithm is developed in order to compute the clock biases and drifts and to remove them from the raw measurements.

A different approach is used to compute Galileo PR and PR rate error for E5a frequency, due to the lack of GPS L5 measurements. For the analysis on E5a measurements, only Galileo measurements are used and the state vectors became:

$$
\begin{aligned}
& \underline{x}=\left[c d t_{u}^{\mathrm{GAL}}\right] \\
& \underline{v}=\left[c \dot{d}_{u}^{\mathrm{GAL}}\right]
\end{aligned}
$$

where $c d t_{u}^{\mathrm{GAL}}$ and $c \dot{d} t_{u}^{\mathrm{GAL}}$ are the bias and the drift between receiver time and GST. E5a raw PR and PR rate measurements are corrected for the satellite clock errors, relativistic effects, the Sagnac and atmospheric effects as for 
E1. After computing the corrected PRs and PR rates, their residual error is defined as:

$$
\begin{aligned}
E_{\mathrm{PR}} & =\rho_{c}-d \\
E_{\mathrm{PR} \text { rate }} & =\dot{\rho}_{c}-\dot{d}
\end{aligned}
$$

where $\dot{d}$ is the projection of the satellite velocity along the satellite-receiver direction; $d$ and $\dot{d}$ are obtained computing satellites position and velocity starting from the satellite ephemeris and the known receiver position.

Since most of the error sources related to the signal propagation have been sensitively reduced, $P R$ and $P R$ rate errors only contain residual biases due to the signal and its transmission.

In order to determine the thermal noise contribution, it is possible to use two receivers connected to the same antenna in a zero-baseline configuration and consider single differences (SDs). SDs are defined as follows:

$\mathrm{SD}=\rho^{R 1}-\rho^{R 2}=c d t_{R 1-R 2}+\varepsilon_{\mathrm{SD}}$

where $\rho^{R 1}$ and $\rho^{R 2}$ are the PRs from the two receivers; $c d t_{R 1-R 2}$ is the difference between the two receiver clock, and $\varepsilon_{S D}$ is the residual error. $c d t_{R 1-R 2}$ is estimated using all the satellites available, and it is removed to analyze the residual error, $\varepsilon_{S D}$. Note that single differencing removes all the common systematic errors.

\section{Experimental setup}

In order to collect Galileo and GPS observables, a Javad RingAnt-G3T was mounted on the rooftop of the European Microwave Signature Laboratory (EMSL) in the Joint Research Centre (JRC) premises in Ispra, Italy. The EMSL, which is the highest building in the area was selected in order to minimize the amount of multipath received by the antenna.

The antenna was then fed to a Septentrio PolarRxS receiver able to simultaneously collect GPS, GLONASS and Galileo measurements on several GNSS bands. Both Galileo $\mathrm{E} 1$, tracked considering the $\mathrm{BOC}(1,1)$ modulation, and E5a signals are considered, and a comparison between measurements from the two frequencies is provided. A second Septentrio PolarRxS receiver was used for SD processing.

In order to verify the hypothesis of absence of multipath, data collected using the PolarRxS receiver were processed using the translating, editing and quality checking (TEQC) software (Estey and Meertens 1999). TEQC allows the analysis of the impact of multipath and ionospheric disturbances at a given site. With respect to multipath, a metric is obtained by combining PR and carrier phase measurements. Large and correlated values of this metric indicate the presence of multipath. In this case, the observed multipath metric assumes low values supporting the hypothesis of reduced multipath.

The result presented below was obtained using a Delay Lock Loop (DLL) bandwidth equal to $0.25 \mathrm{~Hz}$. The same processing parameters were used for GPS and Galileo. The phase lock loop (PLL) bandwidth was set to $15 \mathrm{~Hz}$ for all the signals. The integration time was set to $10 \mathrm{~ms}$ (default value in the Septentrio PolarRxS receiver).

The position of the antenna was carefully surveyed using double difference carrier phase positioning. This information was in turn used to compute $d$ and $\dot{d}$.

With this calibrated setup, it was possible to collect several days of data which were used for the characterization of Galileo observables discussed in the following.

\section{Experimental results: Galileo observables}

One week of data, on E1 and E5a frequencies, was used for the $\mathrm{PR}$ and PR rate analysis, and results relative to IOV satellite vehicle $(\mathrm{SV})$ are presented below. $\mathrm{PR}$ and $\mathrm{PR}$ rate errors are analyzed in terms of RMS, mean and maximum values. Measurements from the E1 frequency are considered as first; a comparison of the PR errors of the four IOVs is shown in Fig. 3; the mean and the standard deviation of the PR errors are depicted as a function of satellite elevation and signal carrier to noise power spectral density $\left(C / N_{0}\right)$. For the satellite elevation, a mask angle of ten degrees is adopted, whereas for the $C / N_{0}$, values, lower than $35 \mathrm{~dB}-\mathrm{Hz}$ are discarded.

The behavior of the PR error is similar for the four IOVs: the error decreases when satellite elevation and $C / N_{0}$ increase; the colored bars represent the mean of the errors, while the standard deviation (STD) is represented by the black lines. The mean error reaches a maximum value of $0.50 \mathrm{~m}$ for the IOV with PRN 19. The error statistics for the four IOVs are summarized in Table 1.

The maximum error varies from $1.86 \mathrm{~m}$, for satellite 12 , to $2.47 \mathrm{~m}$ for satellite 19 ; the RMS values are similar for all satellites with a difference of less than $10 \mathrm{~cm}$. In order to verify the impact of the thermal noise, the performance of the four IOVs is analyzed using SD. In Fig. 4, the RMS of the SD error for the four IOVs is plotted as a function of the $C / N_{0}$.

As for the PR, the SD error behavior of the four IOVs is similar; RMS value decreases passing from 14 to $5 \mathrm{~cm}$ increasing the $C / N_{0}$. Note that the RMS value of the SD error has been normalized by a factor $\sqrt{2}$ to account for the noise amplification due to the pseudorange differencing. Figure 4 quantifies the thermal noise contribution to the total RMS error. This contribution is less than a quarter of the total error budget.

An analysis similar to that carried out on the PRs is performed on the PR rate measurements, and the mean and 
Fig. 3 Galileo E1 PR error as function of satellite elevation and $C / N_{0}$
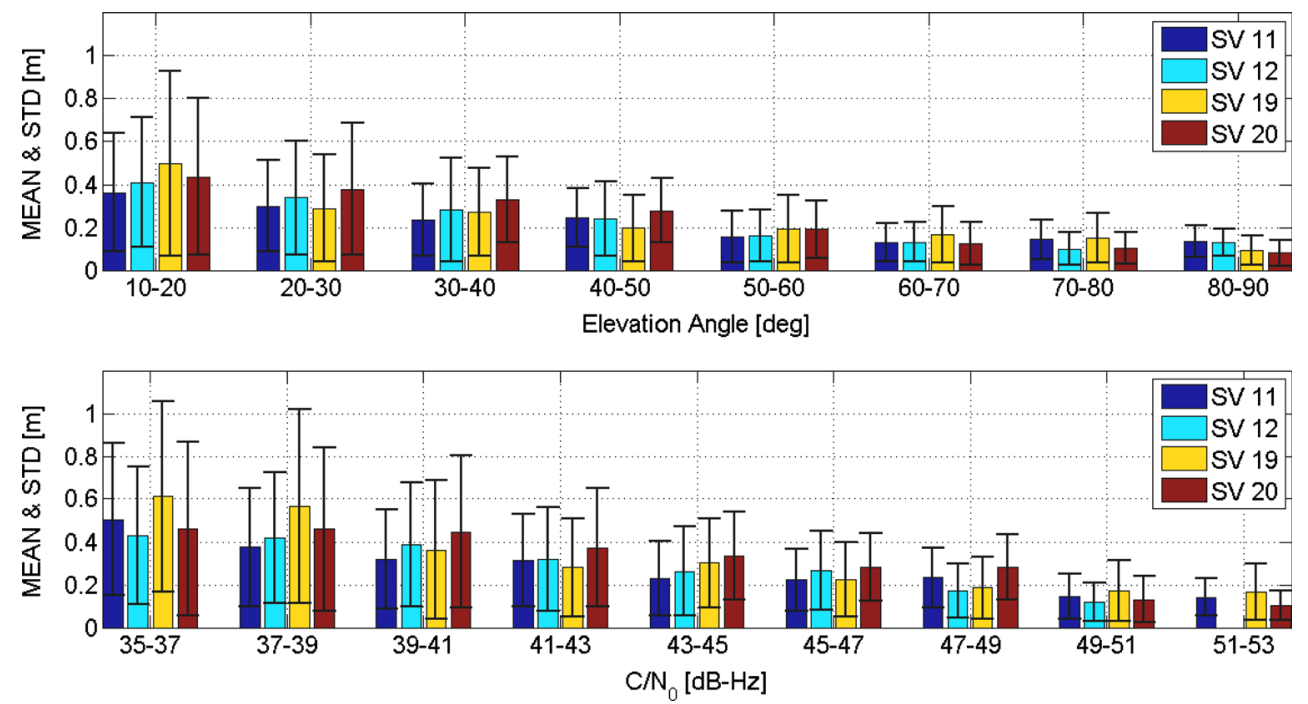

Table 1 IOV E1BC PR error statistics

\begin{tabular}{lll}
\hline PRN & Max (m) & RMS (m) \\
\hline 11 & 2.12 & 0.31 \\
12 & 1.86 & 0.35 \\
19 & 2.47 & 0.37 \\
20 & 2.29 & 0.35 \\
\hline
\end{tabular}

standard deviation of the PR rate errors are shown in Fig. 5 as a function of satellite elevation and $C / N_{0}$.

As for the PRs, the error decreases when the satellite elevation and signal $C / N_{0}$ increase. The error mean reaches a maximum value of $0.0162 \mathrm{~m} / \mathrm{s}$ for IOV with PRN 11 with a STD $0.0125 \mathrm{~m} / \mathrm{s}$. The error statistics for the four IOVs are summarized in Table 2.

The maximum error varies from $0.0741 \mathrm{~m} / \mathrm{s}$, for satellite 19 , to $0.0955 \mathrm{~m} / \mathrm{s}$, for satellite 12 ; the RMS values are similar for all satellites with a difference of less than $2 \mathrm{~mm} / \mathrm{s}$.

In order to obtain a more accurate evaluation of Galileo performance in the E1 frequency, a comparison between Galileo and GPS PR error is performed. The PR error mean and STD are plotted as a function of the satellite elevation and $C / N_{0}$ in Fig. 6.

The height of the bars provides an immediate representation of the magnitude of the mean error and allows a simple comparison between GPS and Galileo. The values relative to Galileo errors are almost halved with respect to GPS. For instance, in the GPS case, the mean error reaches a maximum value of $0.88 \mathrm{~m}$ (for an elevation of $15^{\circ}$ ), whereas for Galileo, this value is limited to $0.50 \mathrm{~m}$. Error statistics such as the maximum and RMS values relative to GPS and Galileo PR errors are summarized in Table 3. The values in Table 3 are obtained by considering all the measurements without accounting for the satellite elevation or $C / N_{0}$.
Fig. 4 Galileo E1 SD error as function of $C / N_{0}$

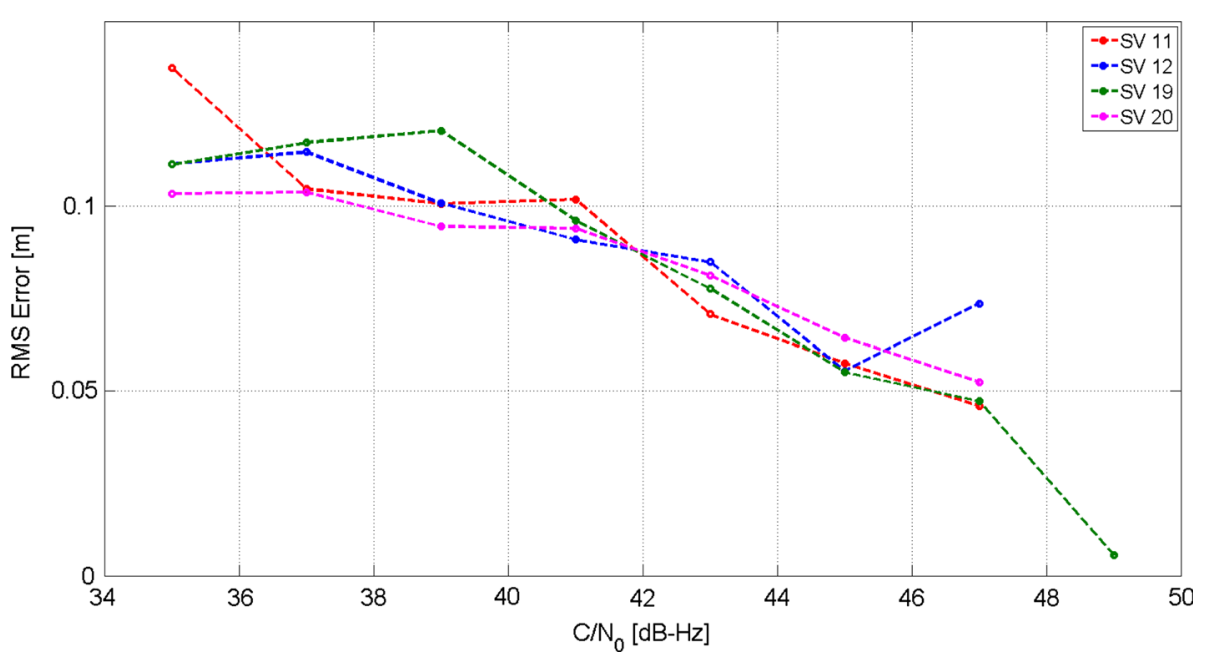


Fig. 5 Galileo E1BC PR rate error as function of satellite elevation and $C / N_{0}$
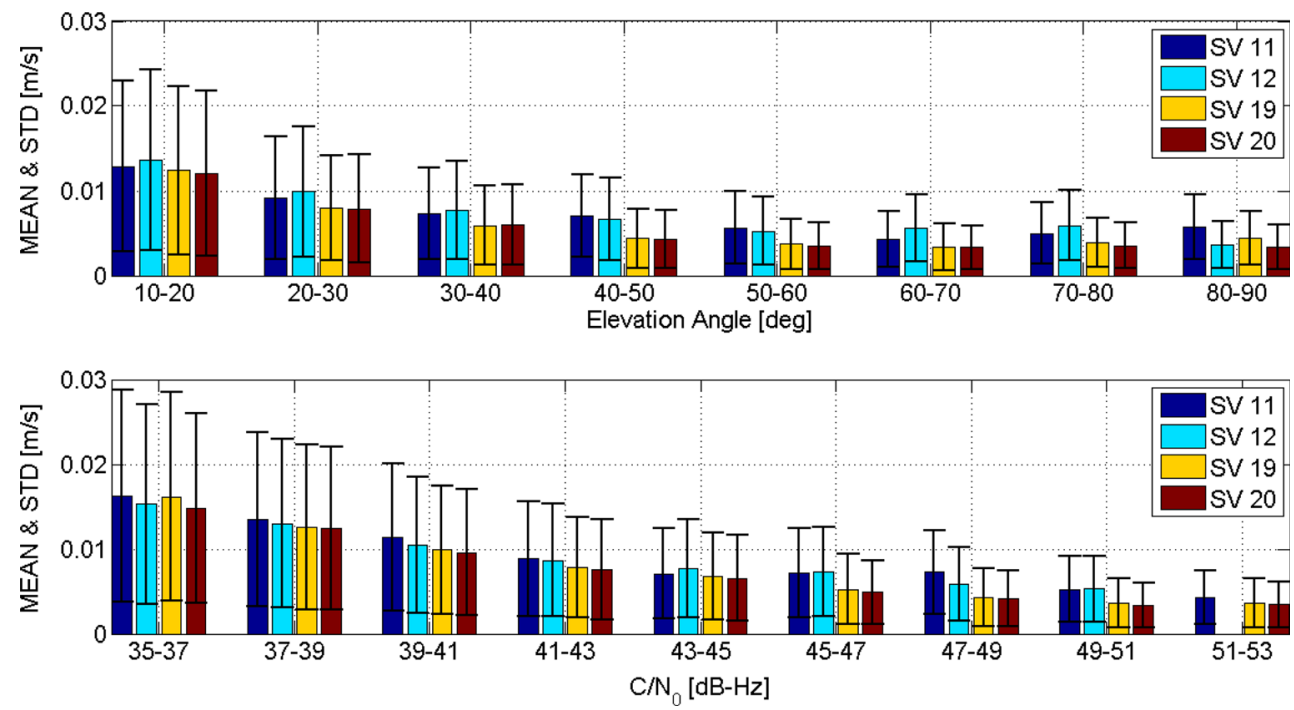

Table 3 GPS (L1) and Galileo (E1BC) PR error statistics error statistics

\begin{tabular}{lll}
\hline PRN & Max $(\mathrm{m} / \mathrm{s})$ & RMS (m/s) \\
\hline 11 & 0.0911 & 0.010 \\
12 & 0.0955 & 0.011 \\
19 & 0.0741 & 0.009 \\
20 & 0.0946 & 0.008 \\
\hline
\end{tabular}

From Table 3, it clearly emerges that Galileo PR errors are smaller than those of GPS. The results obtained are in line with the experimental results presented by (Engel 2008) on GIOVE-B signals and theoretically predicted by (Irsigler et al. 2004) and (Betz 1999).

Analogously, GPS and Galileo PR rate errors are compared as a function of the satellite elevation and $C / N_{0}$ in Fig. 7.

\begin{tabular}{lll}
\hline System & Max (m) & RMS (m) \\
\hline GPS & 4.49 & 0.84 \\
Galileo & 2.47 & 0.34 \\
\hline
\end{tabular}

The two systems are characterized by similar PR rate errors. The Galileo improvement in terms of PR rates is less evident than in the PR case. This fact clearly emerges from Table 4 which summarizes the statistics of PR rate errors considering all the measurements.

The main improvement in PR rate error is related to the maximum error, which is reduced to $9 \mathrm{~cm} / \mathrm{s}$ from the $27 \mathrm{~cm} / \mathrm{s}$ observed in the GPS case.

Galileo PR and PR rate errors were also analyzed for the measurements obtained from the E5a frequency; the
Fig. 6 Galileo (E1BC) and GPS (L1) PR error as function of satellite elevation and $C / N_{0}$
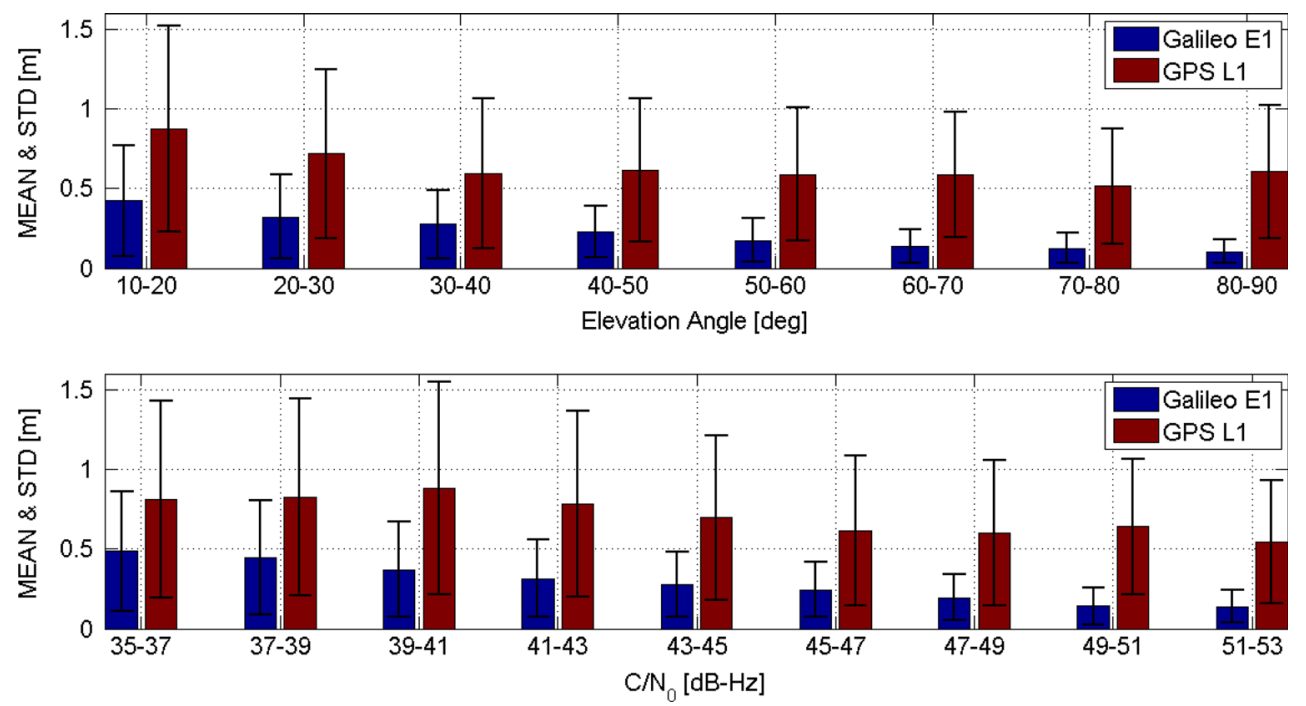
Fig. 7 Galileo (E1BC) and GPS (L1) PR rate error as function of satellite elevation and $C / N_{0}$
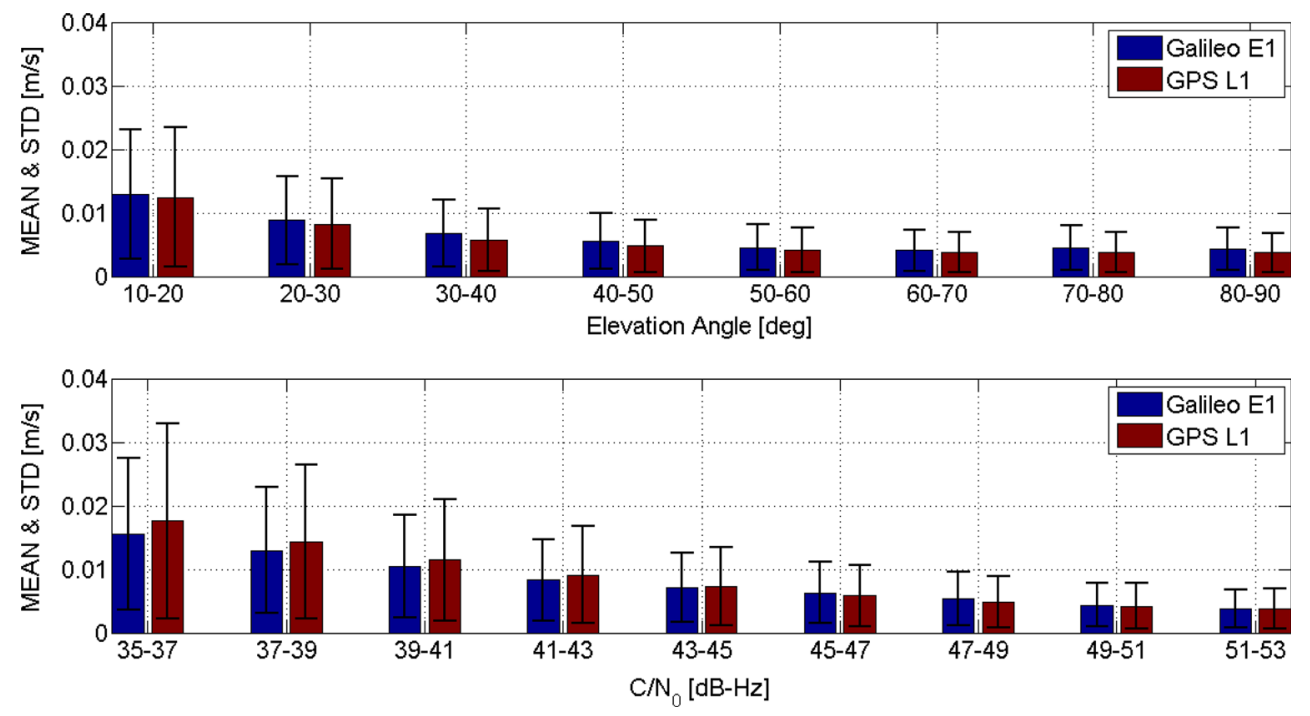

one meter when moving from E5a to E1BC. The RMS error is reduced by $15 \mathrm{~cm}$. This degradation of the E5a signal was not expected, but a similar phenomenon was observed by (Simsky et al. 2008) and (Simsky et al. 2007) for GIOVE-A measurements. The cause of this slight degradation could be the presence of residual ionospheric errors which are 1.8 times larger on E5a than on E1 (Rodriguez et al. 2004).

The same analysis was performed for the PR rate errors, and the results obtained are shown in Fig. 9.

The PR rate errors obtained from the two frequencies are characterized by similar performance, as summarized by the PR error statistics detailed in Table 6.

The advantages of E1BC in this case are less evident; the maximum PR rate error is reduced only by $1 \mathrm{~cm} / \mathrm{s}$, and the RMS value passes from $10 \mathrm{~mm} / \mathrm{s}$ to $11 \mathrm{~mm} / \mathrm{s}$. performance similar to that of the E1BC signal was observed; the maximum PR error is reduced by more than

Fig. 8 Galileo E1BC and E5a PR error as function of satellite elevation and $C / N_{0}$
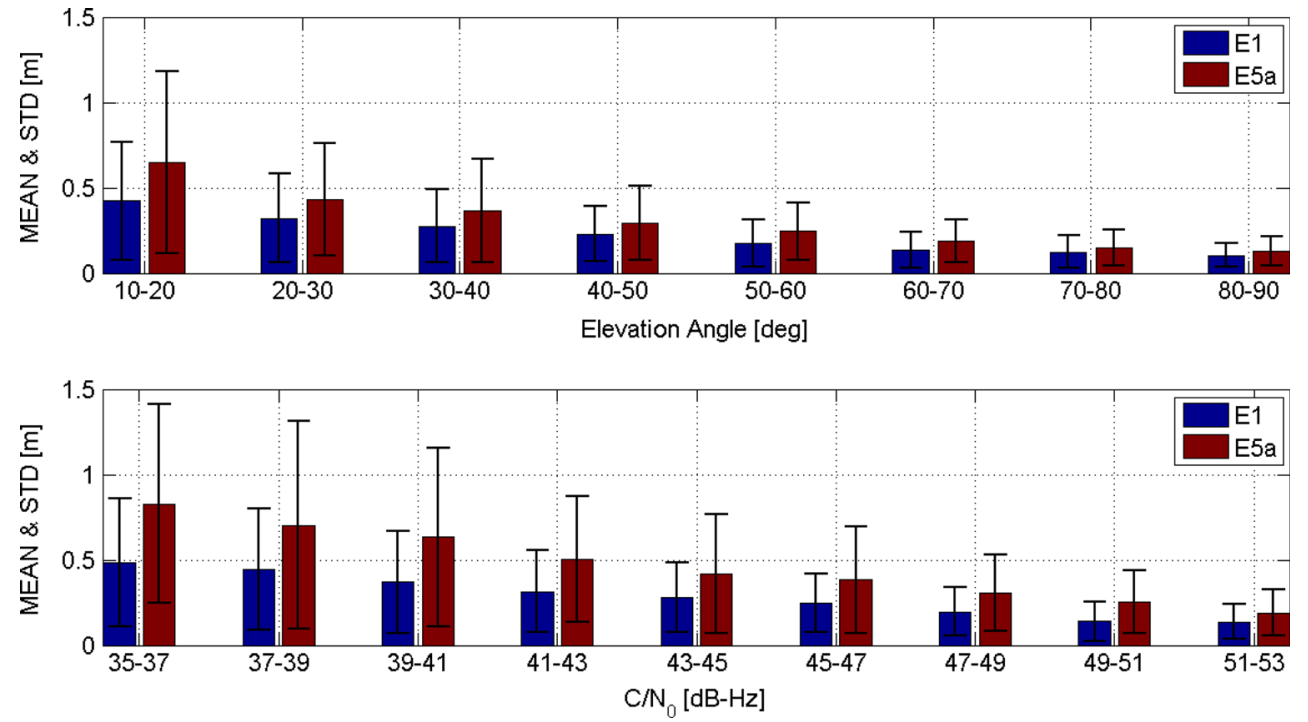
Fig. 9 Galileo E1BC and E5a $\mathrm{PR}$ rate error as function of satellite elevation and $C / N_{0}$
Table 5 E1BC and E5a PR Error Statistics

Table 6 E1BC and E5a PR rate error statistics
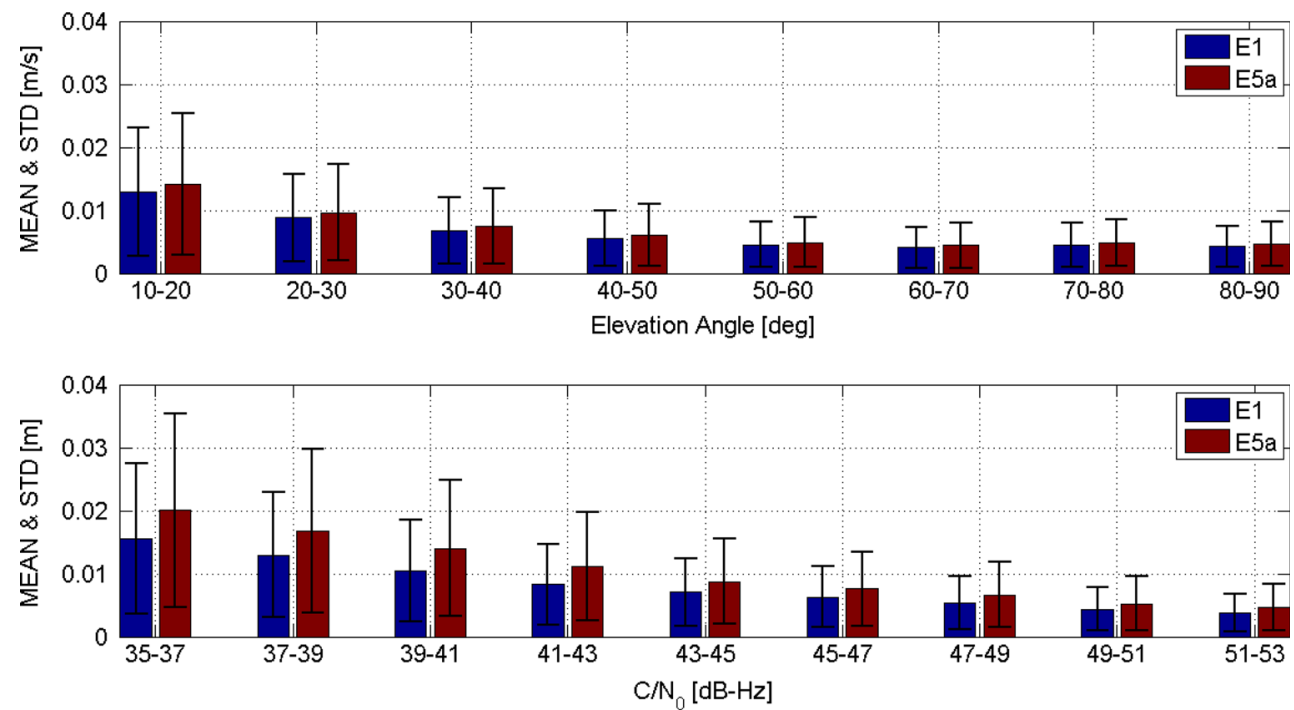

\begin{tabular}{lll}
\hline Frequency & $\begin{array}{l}\text { Max } \\
(\mathrm{m})\end{array}$ & $\begin{array}{l}\text { RMS } \\
(\mathrm{m} / \mathrm{s})\end{array}$ \\
\hline E1 & 2.47 & 0.34 \\
E5a & 3.80 & 0.49 \\
\hline
\end{tabular}

\begin{tabular}{lll}
\hline Frequency & $\begin{array}{l}\text { Max } \\
(\mathrm{m} / \mathrm{s})\end{array}$ & $\begin{array}{l}\text { RMS } \\
(\mathrm{m} / \mathrm{s})\end{array}$ \\
\hline E1 & 0.0955 & 0.010 \\
E5a & 0.1097 & 0.011 \\
\hline
\end{tabular}

\section{PVT analysis}

In order to analyze Galileo-only positioning performance, broadcast ephemerides are used. The Galileo single-point solution is at first compared with the solution obtained using GPS-only measurements. Then the benefits of the inclusion of Galileo measurements are evaluated computing a combined solution using GPS and Galileo together. Position and velocity performance is analyzed in terms of RMS and maximum error for horizontal and vertical components.

One week of data (GPS week 1744) were used for PVT analysis; data were collected at $1 \mathrm{~Hz}$ rate. Note that the week considered is different from the one adopted in (Angrisano et al. 2013), which analyzed data from GPS week 1725 . For this reason, the results presented here are complementary to those analyzed in (Angrisano et al. 2013).

In order to perform a fair comparison between GPS and Galileo, similar geometry conditions are considered and the GPS satellite geometry is artificially degraded. In particular, the following approach is adopted. The Galileo-only solution and its satellite geometry are evaluated. GPS

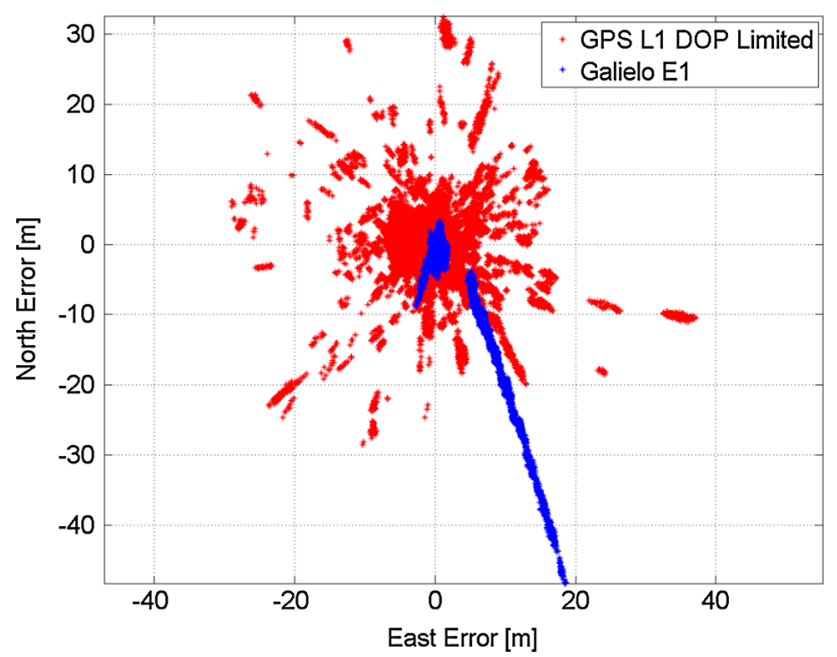

Fig. 10 Horizontal position errors for GPS with limited DOP and Galileo

satellites are then progressively excluded such that a geometry value similar to the Galileo one is obtained. This process is repeated for each epoch analyzed. The parameter selected to quantify the geometry is the horizontal dilution of precision (HDOP); hence, a fair comparison between the two systems is possible for the horizontal component.

Horizontal position errors for Galileo E1BC and GPS (with a limited DOP) are shown in Fig. 10.

In order to present a fair comparison, the two solutions are analyzed in the same epochs, i.e., during those epochs when the four Galileo satellites are available.

The spread of the clouds provides an immediate representation of the magnitude of the error and allows a simple comparison between GPS and Galileo performance. It clearly emerges that the Galileo cloud (blue dots) is significantly reduced with respect to the GPS one (red dots). 
Fig. 11 Horizontal position error (upper box), HDOP values (middle box) and HDOP differences (lower box) as function of time
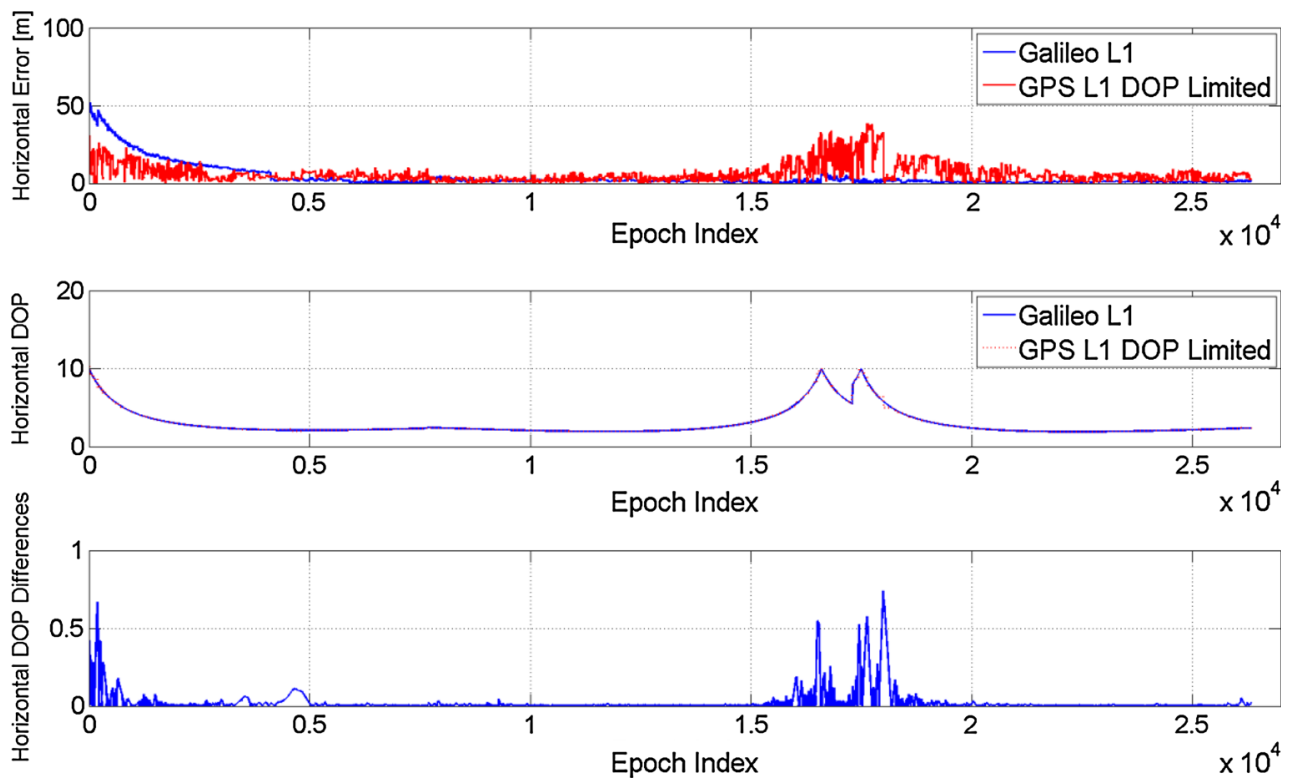

In order to further investigate the performance of the aforesaid configurations, horizontal position errors (upper box), HDOP values (middle box) and difference between HDOP using Galileo and HDOP using GPS (lower box) are plotted as a function of time in Fig. 11.

From Fig. 11, it is possible to observe that the Galileo horizontal position error (blue line) is higher than the GPS one (red line) only during the initial phase when the HDOP is higher than 3 . This error corresponds to the linear trend observed in Fig. 10 where the Galileo estimated position is far away from the central cloud corresponding to the correct position.

Statistical parameters, such as maximum and mean of the horizontal position error, are summarized in Table 7.

From Table 7, it emerges that in average Galileo provides a significant reduction in the position error. In particular, the mean position error is reduced from $6 \mathrm{~m}$ to $4 \mathrm{~m}$. The maximum Galileo error is determined by the linear trend discussed above and is essentially due to a poor geometry. For this reason, the Galileo maximum position error exceeds the corresponding GPS value.

After demonstrating the potentiality of Galileo E1BC signal, through the comparison with GPS, tests in the position domain using E5a measurements and Iono-free E1BC-E5a combination are described. Iono-free measurements are obtained by linear combination of the measurements from the E1BC and E5a frequencies (HoffmannWellenhof et al. 1992, Kaplan and Hegarty 2006):

$\rho_{\mathrm{IF}}=\frac{f_{1}^{2} \rho_{1}-f_{5}^{2} \rho_{5}}{f_{1}^{2}-f_{5}^{2}}$

where $\rho_{\mathrm{IF}}$ is the Iono-free observable; $f_{1}$ and $f_{5}$ are the E1 and E5a center frequencies; and $\rho_{1}$ and $\rho_{5}$ are the PRs from E1 and E5a.
Table 7 GPS limited DOP and Galileo horizontal position error statistics

\begin{tabular}{lll}
\hline Configuration & $\begin{array}{l}\text { Max } \\
(\mathrm{m})\end{array}$ & $\begin{array}{l}\text { Mean } \\
(\mathrm{m})\end{array}$ \\
\hline $\begin{array}{l}\text { GPS limited } \\
\text { DOP }\end{array}$ & 38.57 & 6.03 \\
Galileo & 51.71 & 3.99 \\
\hline
\end{tabular}

Details relative to the Galileo position error evaluated for the three configurations considered are provided in Fig. 12.

The three configurations considered provide similar performance in the position domain for both horizontal and vertical components. Iono-free combination is characterized by the lowest mean horizontal error $(3.88 \mathrm{~m})$, but its maximum error is also the biggest. This is expected since the removal of the first order component of the ionospheric delay in Iono-free combinations is compensated by the amplification of the other measurement errors which are combined ( $\mathrm{Xu}$ 2003). For example, multipath could be amplified by a factor of 3 with respect to single frequency measurements. The same behavior is observed for the vertical component. Statistics relative to the horizontal and vertical components for the considered configurations are summarized in Table 8.

In order to evaluate the benefits of the inclusion of Galileo measurements, a combined solution is performed using GPS and Galileo measurements according to the methodology outlined in Fig. 1. The performance of the multi-constellation solution is compared with the GPS-only case, and the comparison is carried out in the position domain in terms of mean and maximum error for both horizontal and vertical components.

The joint solution is computed considering the Galileo satellites and including the GPS/Galileo time offset as an 
Fig. 12 Horizontal and vertical errors of the Galileo position considering E1, E5a and Ionofree measurements
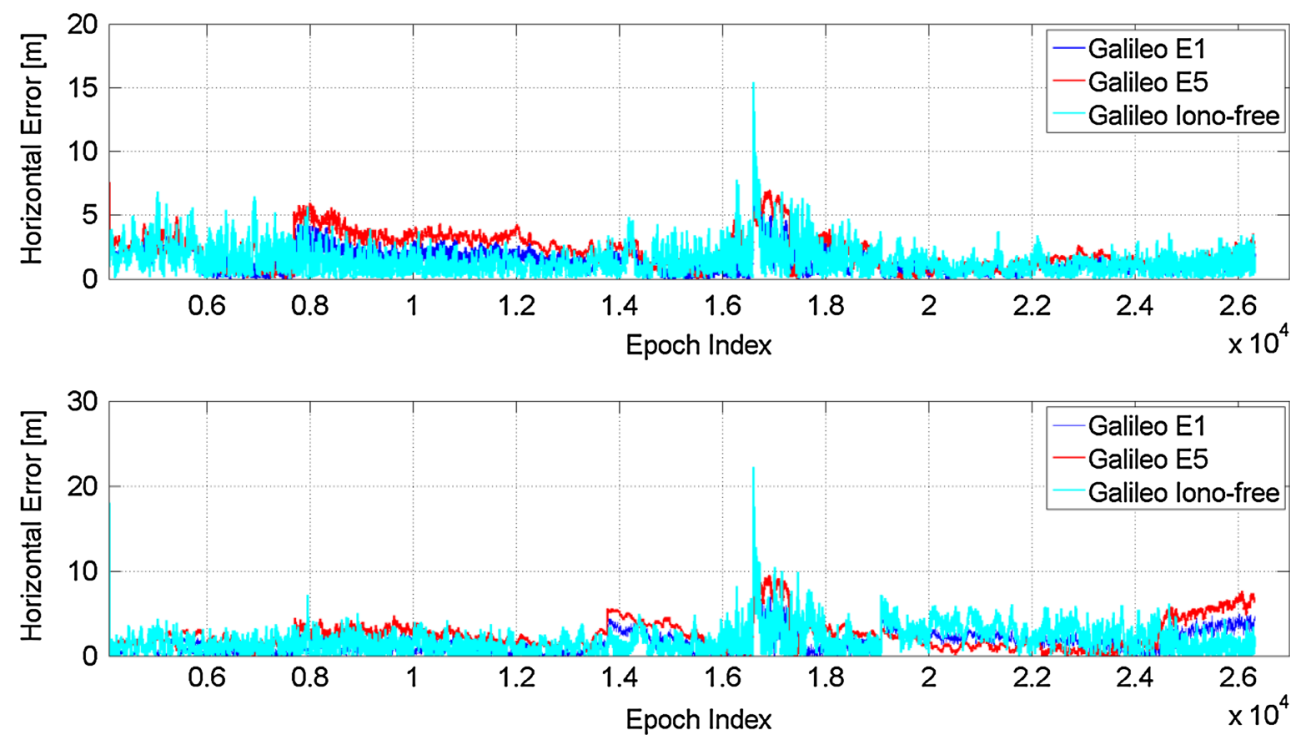

Table 8 Horizontal and vertical position error statistics for E1, E5a and Iono-free combination

\begin{tabular}{llllll}
\hline Configuration & \multicolumn{2}{l}{ Horizontal } & & \multicolumn{2}{l}{ Vertical } \\
\cline { 2 - 3 } \cline { 6 - 6 } \cline { 6 - 6 } & Max $(\mathrm{m})$ & Mean $(\mathrm{m})$ & & Max $(\mathrm{m})$ & Mean $(\mathrm{m})$ \\
\hline Single frequency E1 & 51.71 & 3.99 & & 59.54 & 5.36 \\
Single frequency E5a & 49.56 & 4.68 & & 57.37 & 6.19 \\
Iono-free E1/E5a & 57.87 & 3.88 & & 64.76 & 5.48 \\
\hline
\end{tabular}

additional unknown as shown in (3). The two solutions are analyzed during those epochs when at least one Galileo satellite is available. In this case, however, no real advantage is found since this measurement is used to evaluate the GPS/Galileo time offset. The horizontal position error of the considered configurations is plotted as function of time in the upper box of Fig. 13, whereas the vertical error is provided in the lower part of Fig. 13.

The two configurations are characterized by similar performance; the inclusion of Galileo measurements seems to provide only a slight improvement for both horizontal and vertical components compared with the GPS-only case. The position error statistics are summarized in Table 9, which shows that the mean values of GPS/Galileo horizontal errors is reduced by only $10 \mathrm{~cm}$ with respect to the GPS-only configuration. Also, the vertical mean error is reduced by only $2 \mathrm{~cm}$.

The inclusion of the Galileo observations contributes to a reduction in the maximum error for both horizontal and vertical components; the maximum horizontal error is
Fig. 13 GPS and GPS/Galileo Position error as function of time
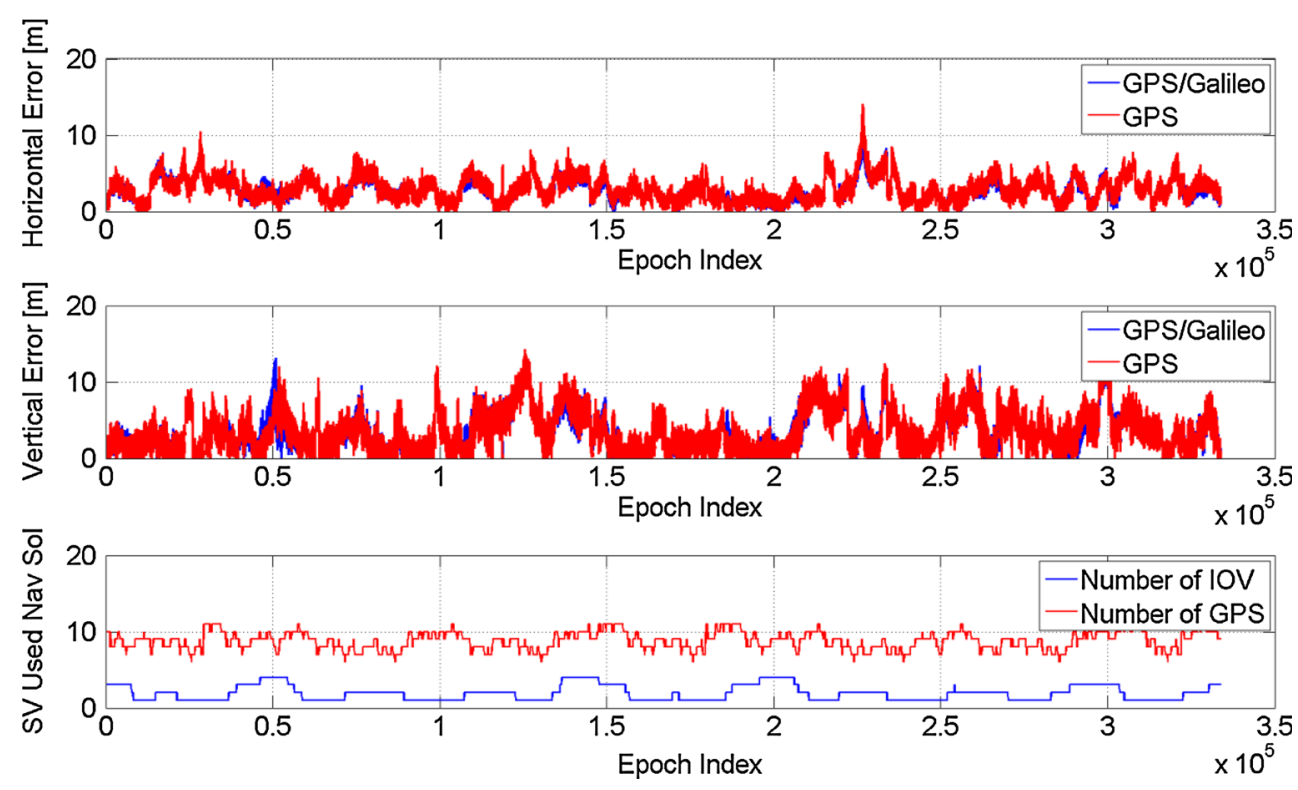
Table 9 Horizontal and vertical position error statistics for GPS, GPS/Galileo combination

\begin{tabular}{llllll}
\hline Configuration & \multicolumn{2}{l}{ Horizontal } & & \multicolumn{2}{l}{ Vertical } \\
\cline { 2 - 3 } & Max $(\mathrm{m})$ & Mean $(\mathrm{m})$ & & Max $(\mathrm{m})$ & Mean $(\mathrm{m})$ \\
\hline GPS & 14.12 & 2.94 & & 14.78 & 3.50 \\
GPS/Galileo & 11.06 & 2.85 & & 14.23 & 3.48 \\
\hline
\end{tabular}

reduced by $3 \mathrm{~m}$ passing from $14 \mathrm{~m}$ in GPS-only case to $11 \mathrm{~m}$ for the multi-constellation case.

\section{Velocity solution}

In this section, results obtained for the analysis of the velocity solution determined using Galileo-only observables are detailed; the velocity solution is compared with the one obtained using GPS with limited HDOP in Fig. 14.

In Fig. 14, the horizontal and vertical components of the velocity estimates are provided separately. Although GPS seems to provide the best velocity solution, the difference between the two systems is less than $2 \mathrm{~cm} / \mathrm{s}$. Statistics relative to the velocity horizontal and vertical components for the configurations considered, including the velocity estimated from Galileo E5a measurements, are summarized in Table 10.

From Table 10, it clearly emerges that the three configurations are characterized by similar performance and the differences are of $\mathrm{cm} / \mathrm{s}$ order. The significant degradation in the vertical component of the GPS velocity solution is due to the rejection of satellites fundamental for the vertical geometry.

As for the position, in order to evaluate the benefits of the inclusion of Galileo PR rate measurements, a combined
Table 10 Horizontal and vertical velocity error statistics for GPS limited DOP, Galileo E1 and E5a

\begin{tabular}{llllll}
\hline Configuration & \multicolumn{3}{l}{ Horizontal } & & \multicolumn{2}{l}{ Vertical } \\
\cline { 2 - 3 } \cline { 6 - 7 } & $\begin{array}{l}\text { Max } \\
(\mathrm{m} / \mathrm{s})\end{array}$ & $\begin{array}{l}\text { Mean } \\
(\mathrm{m} / \mathrm{s})\end{array}$ & & $\begin{array}{l}\text { Max } \\
(\mathrm{m} / \mathrm{s})\end{array}$ & $\begin{array}{l}\text { Mean } \\
(\mathrm{m} / \mathrm{s})\end{array}$ \\
\hline GPS limited DOP & 0.30 & 0.019 & & 1.78 & 0.024 \\
Galileo E1 & 0.43 & 0.046 & & 0.50 & 0.042 \\
Galileo E5a & 0.52 & 0.049 & 0.56 & 0.042 \\
\hline
\end{tabular}

solution is performed using GPS and Galileo observables according to the methodology outlined in Fig. 1. The performance of the multi-constellation configuration is compared with the GPS-only case. The comparison is carried out in the velocity domain in terms of mean and maximum error for both horizontal and vertical components.

The joint solution is computed considering the Galileo satellites and including the GPS/Galileo time drift as additional unknown as in (3). The two solutions are analyzed during those epochs when at least one Galileo satellite is available. In this case, however, no real advantage is found since this measurement is used to evaluate the GPS/Galileo time drift. The horizontal velocity error of the configurations considered is plotted as a function of time in the upper box of Fig. 15, while the vertical error is provided in the lower part.

Statistics relative to the horizontal and vertical velocity components for the configurations compared are summarized in Table 11.

From Table 11, it emerges that the two configurations are characterized by similar performance, and the differences are of $\mathrm{mm} / \mathrm{s}$ order for the mean values, whereas the inclusion of the Galileo measurements reduces the maximum error for both horizontal and vertical components.
Fig. 14 Velocity solution obtained using measurements from the 4 Galileo IOVs and the broadcast ephemerides

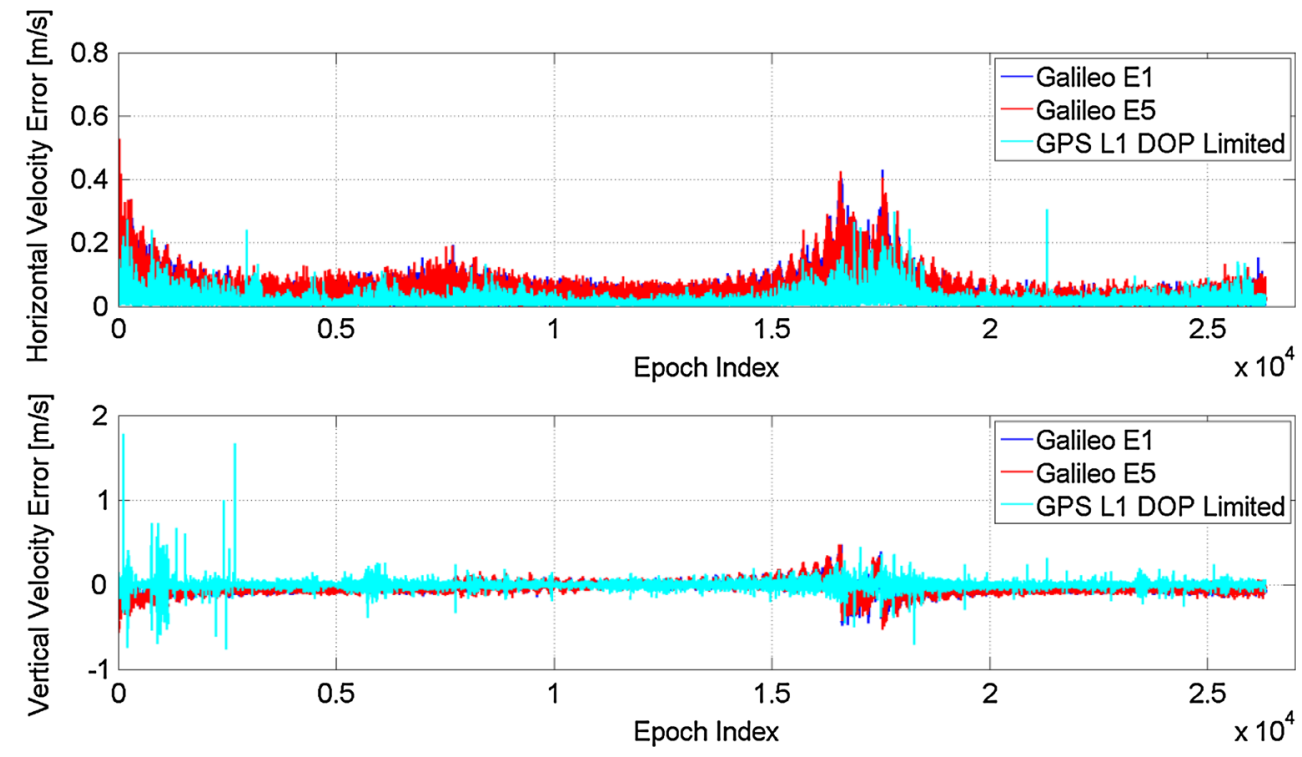


Fig. 15 GPS and GPS/Galileo Velocity error as function of time

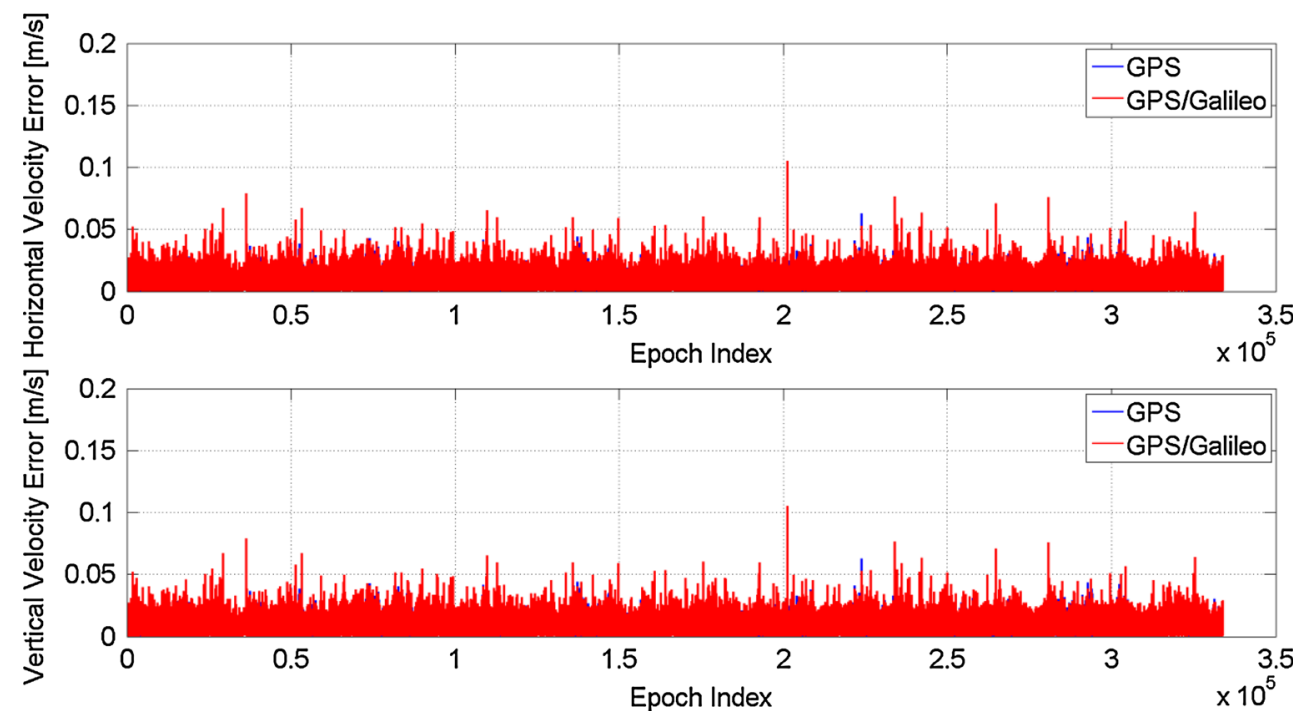

Table 11 Horizontal and vertical velocity error statistics for GPS and GPS $\backslash$ Galileo

\begin{tabular}{llllll}
\hline Configuration & \multicolumn{2}{l}{ Horizontal } & & & Vertical \\
\cline { 2 - 3 } \cline { 6 - 6 } \cline { 5 - 6 } & Max $(\mathrm{m} / \mathrm{s})$ & Mean $(\mathrm{m} / \mathrm{s})$ & & Max $(\mathrm{m} / \mathrm{s})$ & Mean $(\mathrm{m} / \mathrm{s})$ \\
\hline GPS & 0.105 & 0.008 & & 0.098 & 0.002 \\
GPS\Galileo & 0.085 & 0.008 & & 0.066 & 0.001 \\
\hline
\end{tabular}

\section{Conclusions}

The quality of Galileo PRs and PR rates on the E1BC and E5a frequencies was analyzed using precise ephemerides and GIM. The contribution of the thermal noise was isolated using a SD approach.

The performance of Galileo single-point positioning was investigated using broadcast ephemerides, and a comparison between the E1BC and E5a solutions was carried out in the position/velocity domain.

The performance of GPS was used as a term of comparison, and similar geometry conditions were considered; for a fair comparison, the GPS HDOP was degraded to match the Galileo one.

The benefits of the inclusion of Galileo measurements in single-point positioning were studied, comparing the performance of GPS/Galileo multi-constellation with respect to the GPS-only case, for both horizontal and vertical components. Hence, a complete analysis was performed in the measurement and position domains.

The analysis in the PR domain demonstrates that the measurements from the four IOVs are characterized by similar accuracies: for E1BC, the PR RMS error varies from 0.31 to $0.37 \mathrm{~m}$ and the maximum error is of metric order. The analysis on the E1BC and E5a demonstrates that the E5a signal has performance similar to that of the E1BC signal.
For the PR rates, similar conclusions were obtained: The four IOVs provide similar measurement accuracies, and differences are of $\mathrm{mm} / \mathrm{s}$ order. Differences between E1 and E5a measurements are less evident than in the PR case. When compared to GPS, the Galileo PR errors are reduced by almost $50 \%$.

The advantages of the European GNSS clearly emerge in terms of maximum and RMS errors, suggesting that the improved structure of Galileo signals allows a GNSS receiver to extract less noisy measurements. Note that for the analysis, the same processing parameters were used for GPS and Galileo signals.

In the position domain, the comparison between Galileo and GPS with limited HDOP demonstrates the advantage of Galileo: The mean error is reduced of $2 \mathrm{~m}$ in the position domain, whereas in the velocity domain, the considered configurations are characterized by similar performance with differences lower than $2 \mathrm{~cm} / \mathrm{s}$.

A Iono-free solution was also computed using PRs from E1 and E5a. As expected, this solution leads to a reduction in the mean error. Finally, when combining measurements from GPS and Galileo, it was shown that the maximum positioning error is only slightly reduced with respect to the GPS-only case.

Open Access This article is distributed under the terms of the Creative Commons Attribution License which permits any use, distribution, and reproduction in any medium, provided the original author(s) and the source are credited.

\section{References}

Angrisano A, Gaglione S, Gioia C, Robustelli U, Vultaggio M (2012) GIOVE satellites pseudorange error assessment. J Navig 65(1):29-40 The Royal Institute of Navigation, January

Angrisano A, Gaglione S, Gioia C, Borio D, Fortuny-Guasch J (2013) Testing the test satellites: the Galileo IOV measurement 
accuracy. In: Proceedings of the international conference on localization and GNSS (ICL-GNSS 2013 Torino), pp 1-6, June 2013

Betz JW (1999) The offset carrier modulation for GPS modernization. In: Proceedings of the 1999 National Technical Meeting of the Institute of Navigation, San Diego, CA, pp 639-648

Engel U (2008) A theoretical performance analysis of the modernized GPS signals. In: Proceedings of IEEE/ION PLANS 2008, Monterey, CA, May 2008, pp 1067-1078

Estey LH, Meertens CM (1999) TEQC: the multi-purpose toolkit for GPS/GLONASS data. GPS Solut 3(1):42-49

Hoffmann-Wellenhof B, Lichtenegger H, Collins J (1992) Global positioning system: theory and practice. Springer, New York

Irsigler M, Hein GW, Eissfeller B (2004) Multipath performance analysis for future GNSS signals. In: Proceedings of the 2004 national technical meeting of the institute of navigation, San Diego, CA, January 2004, pp 225-238

IS-GPS-200 (2004) Navstar GPS space segment/navigation user interfaces, Revision D. ARINC Research Corporation, El Segundo, CA

Kaplan ED, Hegarty J (2006) Fundamentals of satellite navigation. In: Kaplan ED (ed) Understanding GPS: principles and applications, 2nd edn. Artech House Mobile Communications Series, London

OS-SIS-ICD (Feb 2010) European GNSS (Galileo) open service, signal in space interface control document. European Union and European Space Agency (ESA), Tech Rep, Issue 1

Rodriguez JAA, Hein GW, Irsigler M, Pany T (2004) Combined Galileo/GPS frequency and signal performance analysis. In: Proceedings of the ION GNSS 2004 Long Beach California

Simsky A, Mertens D, Sleewaegen J-M, Hollreiser M and Crisci M (2007) Multipath and tracking performance of Galileo ranging signals transmitted by GIOVE-A. In: Proceedings of the 20th international technical meeting of the satellite division of the institute of navigation (ION GNSS 2007) September 25-28, 2007 Fort Worth Convention Center Fort Worth, TX

Simsky A, Mertens D, Sleewaegen J-M, Hollreiser M, Crisci M (2008) Experimental results for the multipath performance of Galileo signals transmitted by GIOVE-A Satellite. Int J Navig Obs vol 2008, Article ID 416380, 13 pp

Steigenberger P, Hugentobler U, Montenbruck O, Hauschild A (2011) Precise orbit determination of GIOVE-B based on the CONGO network. J Geodesy 85(6):357-365

Xu G (2003) GPS: theory, algorithms and applications. Springer, Berlin Heidelberg. ISBN 978-3-540-72714-9

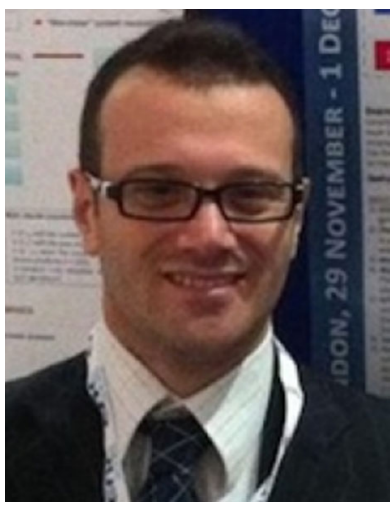

Ciro Gioia received the M.S. degree in Nautical Sciences from Parthenope University, Italy, in 2009. In April 2014 he successfully defended his $\mathrm{PhD}$ thesis at the same University. On 2013 he has been a visiting student at the JRC of the European Commission. Since May 2014 he is external consultant at JRC. His research interests focuses on location and navigation with special emphasis on geomatics aspects.

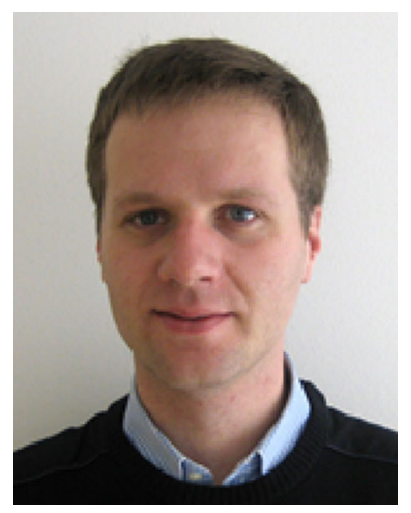

Daniele Borio received the M.S. and the Ph.D. degree from Politecnico di Torino in 2004 and 2008, respectively. From January 2008 to September 2010 , he was a senior research associate in the PLAN group of the University of Calgary, Canada. He is currently a scientific policy officer at the Joint Research Centre of the European Commission in the fields of digital and wireless communications, location and navigation.

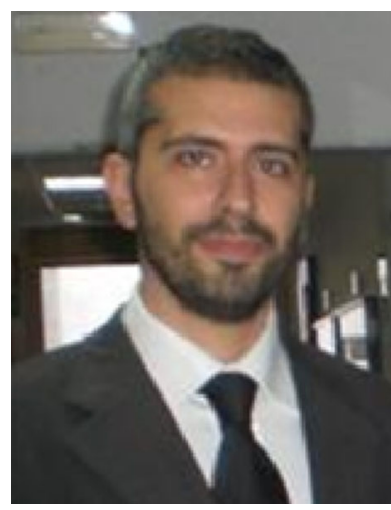

Antonio Angrisano has obtained his MSc degree (Cum Laude) in Science of Navigation at Parthenope University of Naples (2006). He has obtained his PhD on April 2011 in Geodetic and Topographic Sciences at Parthenope University with a dissertation entitled "GNSS/ INS Integration Methods". He is currently a Post-Doc at Parthenope University and his main research interests include GNSS, Inertial Navigation, RAIM.

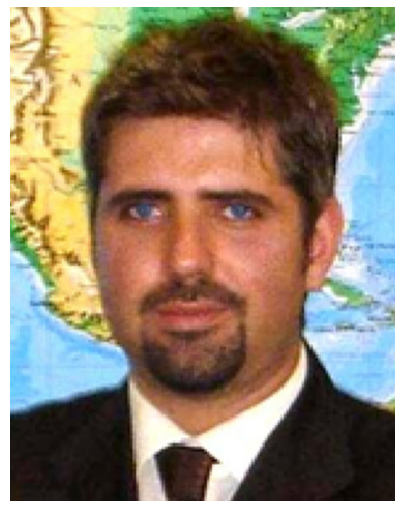

Salvatore Gaglione has been Assistant Professor in Navigation since 2008 at the Science and Technology Department of Parthenope University of Naples. On 2010 he was Visiting Academic at "Department of Geomatics Engineering" of University of Calgary. He is Scientific Director of the Parthenope University "Navigation Laboratory" and Responsible of the Naples EGNOS Data Collection Network station.

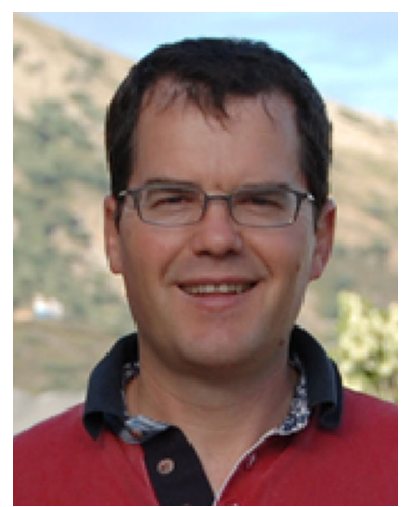

Joaquim Fortuny-Guasch received the M.S. and the Ph.D., respectively, from the Technical University of Catalonia (UPC) and the Universitat Karlsruhe (TH). Since 1993, he has been with the European Commission Joint Research Centre, Ispra, Italy, where he is a senior scientific officer. His current research interests are in the fields of GNSS, spectrum monitoring and geolocation of interference sources. 